\title{
Placental mTOR complex 1 regulates fetal programming of obesity and insulin resistance in mice
}

\author{
Brian Akhaphong, ${ }^{1}$ Daniel C. Baumann, ${ }^{1}$ Megan Beetch, ${ }^{1}$ Amber D. Lockridge, ${ }^{1}$ Seokwon Jo, ${ }^{1}$ \\ Alicia Wong, ${ }^{1}$ Tate Zemanovic, ${ }^{1}$ Ramkumar Mohan, ${ }^{1}$ Danica L. Fondevilla, ${ }^{1}$ Michelle Sia, ${ }^{1}$ \\ Maria Ruth B. Pineda-Cortel, ${ }^{2,3}$ and Emilyn U. Alejandro' \\ 'Department of Integrative Biology \& Physiology, University of Minnesota Medical School, Minneapolis, Minnesota, \\ USA. ${ }^{2}$ Research Center for the Natural and Applied Sciences and ${ }^{3}$ Department of Medical Technology, University of Santo \\ Tomas, Manila, Philippines.
}

Fetal growth restriction, or low birth weight, is a strong determinant for eventual obesity and type 2 diabetes. Clinical studies suggest placental mechanistic target of rapamycin (mTOR) signaling regulates fetal birth weight and the metabolic health trajectory of the offspring. In the current study, we used a genetic model with loss of placental mTOR function (mTOR-KOPacenta) to test the direct role of mTOR signaling on birth weight and metabolic health in the adult offspring. mTOR-KOPlacenta animals displayed reduced placental area and total weight, as well as fetal body weight at embryonic day (E) 17.5. Birth weight and serum insulin levels were reduced; however, $\beta$ cell mass was normal in mTOR-KO Placenta newborns. Adult mTOR-KOPlacenta offspring, under a metabolic high-fat challenge, displayed exacerbated obesity and metabolic dysfunction compared with littermate controls. Subsequently, we tested whether enhancing placental mTOR complex 1 ( $m$ TORC1) signaling, via genetic ablation of TSC2, in utero would improve glucose homeostasis in the offspring. Indeed, increased placental mTORC1 conferred protection from diet-induced obesity in the offspring. In conclusion, placental mTORC1 serves as a mechanistic link between placental function and programming of obesity and insulin resistance in the adult offspring.

Conflict of interest: The authors have declared that no conflict of interest exists.

Copyright: (c) 2021, Akhaphong et al. This is an open access article published under the terms of the Creative Commons Attribution 4.0 International License.

Submitted: March 4, 2021

Accepted: May 19, 2021

Published: July 8, 2021

Reference information: /CI Insight. 2021;6(13):e149271.

https://doi.org/10.1172/jici. insight.149271.

\section{Introduction}

Type 2 diabetes (T2D) affects more than 350 million individuals worldwide (1). It is a complex disease characterized by pancreatic $\beta$ cell failure in the setting of obesity and insulin resistance in the peripheral tissues. Known genetic variants account for less than $10 \%$ of the risk for T2D $(2,3)$. Evidence from human epidemiology as well as animal studies shows that fetal nutrient environmental factors provide significant susceptibility to T2D (2-6). Indeed, adverse maternal influences in pregnancy are linked to alteration in fetal birth weight and the long-term health of the offspring. In the United States, about $8 \%$ of infants are born with low birth weight (7), a proxy for poor fetal growth. Placental insufficiency is the primary cause of fetal growth restriction (FGR) or low birth weight, which are strong determinants for eventual development of obesity and T2D (8).

The placenta senses and responds to changes in the maternal environment by altering its structure and function (e.g., nutrient transport) and matching maternal resources and fetal growth (9). The placenta is thought to integrate maternal signals to placental function through the mechanistic target of rapamycin (mTOR) kinase. mTOR transduces signals from nutrients and growth factors to regulate cellular growth and organ size, but its role in the placenta remains poorly understood. mTOR is highly expressed in the syncytiotrophoblast of the human placenta (10), and its expression and activity are regulated by glucose, amino acids (AAs), and hormones such as insulin and IGF-1. Association-based human studies suggest that fetal placental mTOR signaling may serve as a critical link between the maternal nutrient supply and growth of the developing fetus (11). Possible mechanisms by which mTOR can regulate fetal growth may involve regulation of nutrient transporters in the placenta $(10,12)$ and trophoblast cells' development (13). FGR in human and animal models show reduced concentrations of placental AAs, such as leucine, that are associated with reduced mTOR activity $(10,14-16)$. Downstream of mTOR, the mTOR complex 1 (mTORC1) pathway is negatively regulated by TSC2. MTORC1 is known to affect placental AA transporter activity (16), while mTORC2/Akt signaling regulates trophoblast development (13). Therefore, placental mTOR 
signaling may not only direct placental metabolism and growth but also influence the development and longterm health trajectory of the offspring. However, the role of placental mTOR signaling in fetal programming of metabolic disease has not been directly studied in vivo.

Human studies show that reduced placental mTOR activity is correlated with FGR $(10,17)$, whereas increased activity is positively correlated with fetal overgrowth, such as in maternal obesity (18). Fetal overgrowth, or macrosomia, is an adverse outcome of gestational diabetes mellitus (GDM) and increases the risk of the offspring of developing obesity/T2D in adulthood (19). A causal relationship between placental mTOR and fetal birth weight and long-term health of the offspring has not been established. Thus, a murine model testing the direct role of placental mTOR on fetal programming of metabolic health of the offspring would fill this knowledge gap.

In the present study, we tested the hypothesis that placental mTOR regulates fetal birth weight as well as the development of obesity and T2D in the adult offspring. We used the specific placental CYP19Cre recombinase to genetically delete $\mathrm{mTOR}$ or to increase mTORC1 activity through the deletion of TSC2, the negative regulator of $\mathrm{mTORC} 1$, in trophoblast cells of the placenta. Characterization of the metabolic phenotypes in the offspring at birth and in adulthood, under normal and high-fat diet (HFD) conditions, revealed an increased susceptibility to obesity and glucose homeostasis dysfunction in the adult offspring that experienced loss of placental mTOR (CYP19Cre mTOR $^{\text {flfl}}$, hereafter mTOR-KO $\mathrm{K}^{\text {Placenta }}$ ), whereas gain of placental mTORC1 activity conferred protection under metabolic stress. Our data demonstrate direct evidence for placental mTOR signaling in the regulation of fetal growth and the programming of obesity and T2D. These studies provide a strong rationale to target placental mTOR signaling in FGR-related complications in human pregnancy.

\section{Results}

Generation of genetic placental mTOR ablation model in mice. To understand the impact of placental mTOR on fetal weight and metabolic health, we created a mouse model in which mTOR is genetically deleted in the placenta by utilizing the human CYP19 gene to drive the Cre recombinase transgene starting at embryonic day (E) 6.5 (20), a technique previously shown to effectively induce placenta-specific gene deletion in the mouse $(21,22)$. The schematic diagram of the study is shown in Figure 1A. First, we verified placental specificity of the CYP19Cre using a green fluorescent protein reporter line (CAG-GFP, Figure 1B), where we detected GFP throughout the entire placenta only in CYP19Cre E17.5 embryos. By Western blot, we determined a significant reduction in mTOR protein level, as well as the phosphorylation status of S6 (Ser 240), a downstream target of mTOR, in E15.5 placenta lysates of mTOR-KO ${ }^{\text {Placenta }}$ compared with WT controls (Figure 1C). X-CLARITY-based tissue clearing and spectral imaging in whole bodies of newborns (postnatal day $0, \mathrm{P0}$ ) showed relatively equal low GFP background signal between mTOR-KO ${ }^{\text {Placenta }}$ and WT Ctrl (GFP-, Supplemental Figure 1A; supplemental material available online with this article; https:// doi.org/10.1172/jci.insight.149271DS1), suggesting lack of reporter expression in the whole body of both genotypes (Ctrl and CYP19Cre $e^{+}$CAG-GFP) (22). Supporting the X-CLARITY data, we observed comparable levels of mTOR protein in whole embryonic livers, in addition to visceral fat and islets in adult mTOR-KOPlacenta and Ctrl offspring (Supplemental Figure 1, B-D).

Reduced placental weight and fetal body weight in $m$ TOR-KO Placenta offspring. In human pregnancies, decreased mTOR signaling is associated with decreased birth weight (10). To better understand the impact of placental mTOR loss on embryonic growth, we assessed the weight and architecture of the placenta on E17.5. Representative H\&E images of E17.5 placenta are shown in Figure 1D. Consistent with the known association of placental mTOR signaling and fetal growth, we observed a reduction in placental area (Supplemental Figure 2A). Total placental weight was reduced in mTOR-KO ${ }^{\text {Placenta }}$ animals at E17.5 (Supplemental Figure $2 \mathrm{~B}$ ), and this was significant in female mTOR-KO ${ }^{\text {Placenta }}$ animals (Figure 1E). The LZ mass was smaller, and a trend toward decrease in JZ mass was also observed in E17.5 mTOR-KO ${ }^{\text {Placenta }}(P=0.07$, Supplemental Figure 2, C and D). To control body weight measurement variability in P0 (due to differences in time of delivery), we measured body weight at E17.5. A significant reduction in body weight was observed in E17.5 and P0 mTOR-KO ${ }^{\text {Placenta }}$ (Supplemental Figure 2E). However, significance was observed only in females at E17.5 mTOR-KO ${ }^{\text {Placenta }}$ (Figure 1, F and G). Together, these data show that deletion of placental mTOR reduced body weight of the offspring.

Normal pancreatic $\beta$ cell mass but reduced serum insulin levels in mTOR-KOPlacenta offspring. Pancreas development is sensitive to nutrient availability in utero $(4,23)$. Female mTOR-KO ${ }^{\text {Placenta }}$ mice displayed reduced nonfasted blood glucose (Figure $1 \mathrm{H}$ and Supplemental Figure 2F). Nonfasted serum insulin levels were 
A

Male and Female mTORKO Newborns
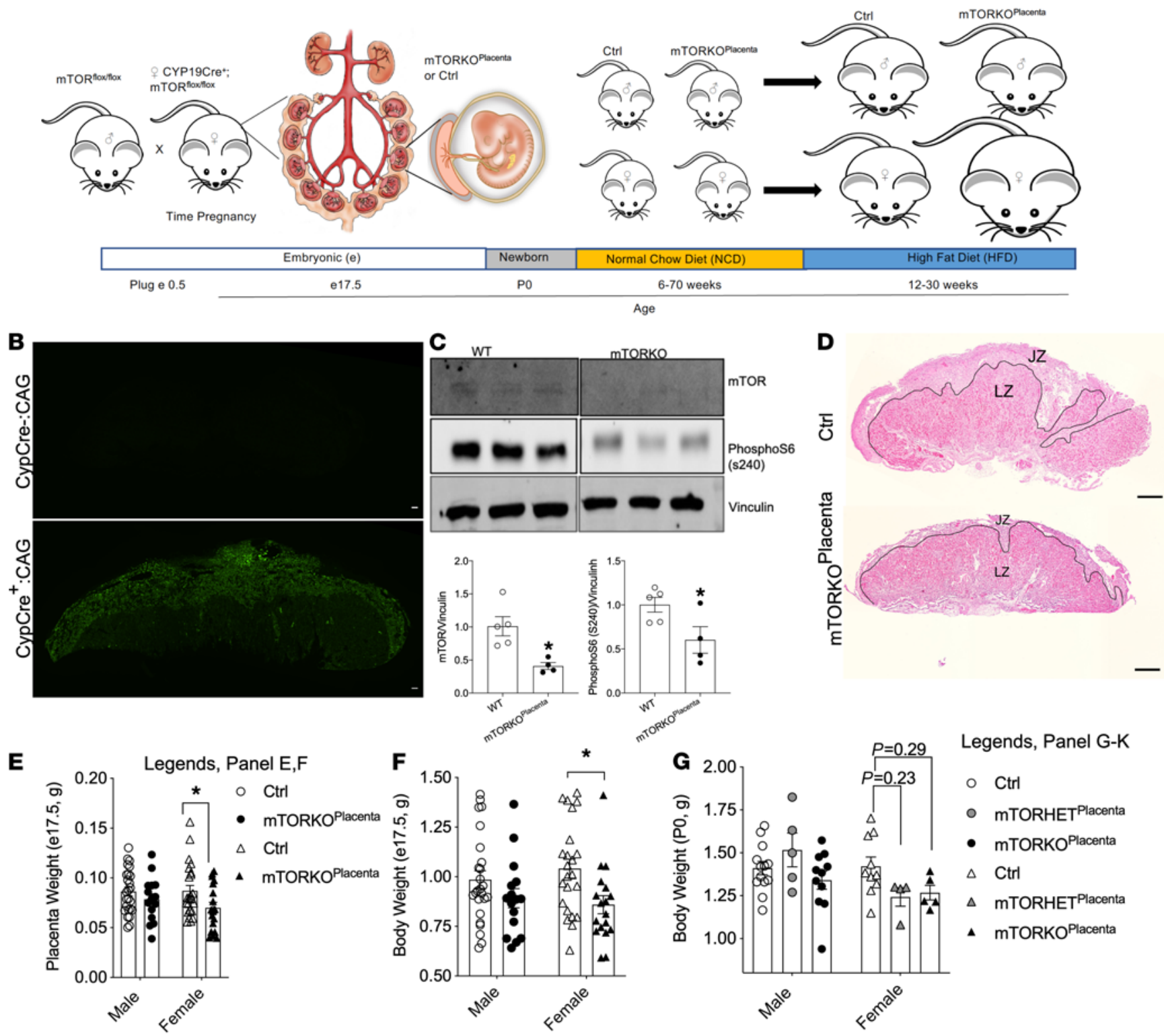

Legends, Panel G-K

H

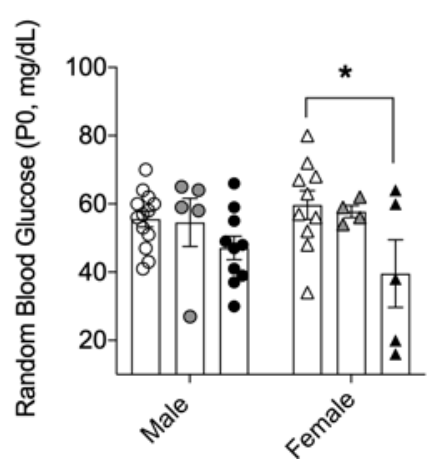

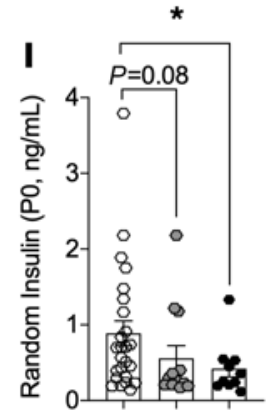

J

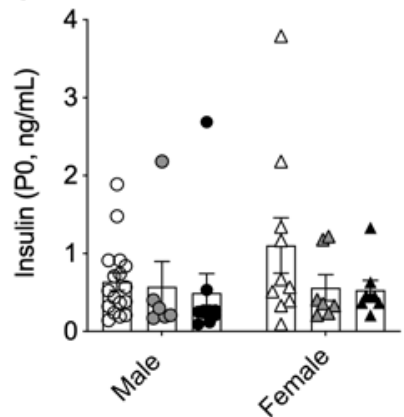

$\mathbf{K}$

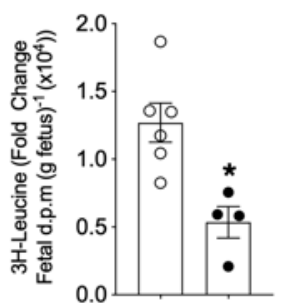

Figure 1. Reduced placental weight and fetal body weight in mTOR-KOPlacenta embryos. Schematic diagram and timeline of the mTOR-KO study (A). Endogenous GFP reporter (CAG-ZsGreen1) in control (CYP19Cre)), positive control (CYP19Cre+) experimental placentas (B). Representative Western blot of E15.5 placenta lysates for mTOR and phosphorylated S6 (Ser 240) and respective quantifications normalized to vinculin (C). H\&E staining of control (Ctrl) and mTOR-KOPlacenta (D, junctional zone [JZ] and labyrinth zone [LZ]). Placental weight of E17.5 males ( $n=25$ Ctrl, 16 mTOR-KO Placenta) and females ( $n=24$ Ctrl, 18 mTOR-KO Placenta, E). E17.5 fetal weight separated by male ( $n=25$ Ctrl, 16 mTOR-KO ${ }^{\text {Placenta) }}$ ) and female $\left(n=23\right.$ Ctrl, 18 mTOR-KO $\left.{ }^{\text {Placenta, }}, \mathbf{F}\right)$. PO (within 12 hours of birth) body weight separated by male ( $n=13 \mathrm{Ctrl}, 5 \mathrm{mTOR}-\mathrm{HET}^{\text {Placenta, }}, 11 \mathrm{mTOR}-\mathrm{KO}{ }^{\text {Placenta) }}$ ) and female ( $n=10$ Ctrl, 4 mTOR-HET Placenta, 5 mTOR-KOPlacenta, G). Random blood glucose was measured from trunk blood of PO males ( $n=13$ Ctrl, 5 mTOR-HET Placenta, 10 mTOR-KO Placenta) and females $(n=10$ Ctrl, 


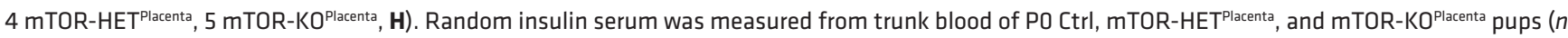
$=25,13,10$, respectively) from 5 dams (I). Random insulin serum was measured from trunk blood of PO Ctrl, mTOR-HET Placenta, and mTOR-KO Placenta males $\left(n=17,6,10\right.$, respectively) and females $\left(n=10,7,9\right.$, respectively) from 5 dams $(\mathrm{J})$. Fetal ${ }^{3} \mathrm{H}$-Leucine uptake after 10 minutes of leucine placental perfusion in utero ( $n=4-6)$ from 3 dams (K). Statistical analysis was performed using 2-tailed Mann-Whitney (C, I, and $\mathbf{K})$ and 2-way ANOVA with Sidak's multiple comparisons (E-G, H, and J). Error bars represent mean \pm SEM. ${ }^{*} P<0.05$ Ctrl vs. mTOR-KOPlacenta. Scale bars in images $(\mathbf{B}$ and $\mathbf{D})$ are $200 \mu \mathrm{m}$.

reduced in mTOR-KO Placenta in P0 animals (Figure 1I). After considering sex as a variable, there were no significant changes observed between groups (Figure $1 \mathrm{~J}$ ). Normal $\beta$ cell mass was detected in both male and female mTOR-KO Placenta mice compared with littermate controls (Supplemental Figure 2, G and H). Collectively, these data show that deletion of placental mTOR reduced insulin levels and decreased glucose, but not $\beta$ cell mass, in the newborn offspring.Reduced leucine transfer from mother to $m$ TOR-KOPlacenta offspring. Placental mTOR has been previously implicated to regulate AA transfer from mother to fetus $(24,25)$. Next we measured plasma AAs in P0 mTOR-KO ${ }^{\text {Placenta }}$ and littermate controls, but we found no differences among all the AAs detected (Supplemental Figure 3A). Since mTORC1 has been shown to regulate system A and system L AA transport in primary human trophoblast cells (12), and leucine transport is downregulated in FGR (10), we specifically measured leucine transfer from the maternal to fetal circulation with or without placental mTOR deletion. Our data showed reduced leucine transport from mothers to embryos of mTOR-KO ${ }^{\text {Placenta }}$ compared with littermate controls (Figure $1 \mathrm{~K}$ ).

Adult male and female mTOR-KO Placenta offspring display normal glucose homeostasis phenotypes. Low birth weight is a strong determinant for eventual metabolic dysfunction in adulthood. To assess the metabolic impact of placental mTOR in adult offspring, we followed offspring from birth to adulthood and performed in vivo phenotyping under normal chow diet (NCD). Fetal programming can have sexually dimorphic effects in the offspring; therefore, metabolic phenotyping was done in both sexes. Male mTOR-KO ${ }^{\text {Placenta }}$ mice displayed a mildly increased body weight starting at week 10 (Supplemental Figure 4A) compared with their littermate controls. Nonfasted blood glucose was normal at 11-20 weeks of age (Supplemental Figure 4B). At 8 weeks of age, male mTOR-KO ${ }^{\text {Placenta }}$ mice displayed normal glucose tolerance (Supplemental Figure 4C) and insulin sensitivity (Supplemental Figure 4D). Comparable body weight (Supplemental Figure 4E) and nonfasted blood glucose at 11-20 weeks of age were observed in female mTOR-KO ${ }^{\text {Placenta }}$ compared with littermate controls (Supplemental Figure $4 \mathrm{~F}$ ). Similarly, female mTOR-KO ${ }^{\text {Placenta }}$ mice displayed normal glucose tolerance and insulin sensitivity at 12 weeks (Supplemental Figure 4, G and $\mathrm{H}$ ).

Adult female $m$ TOR-KO Placenta mice display exacerbated obesity phenotypes under HFD challenge. Sex differences are observed in animal models of fetal programming (26). Therefore, we tested whether female mTOR-KOPlacenta mice are more susceptible to a "second hit" insult, such as HFD. Assessment of body weight throughout the course of HFD treatment revealed significant weight gain in the female mTOR-KO ${ }^{\text {Placenta }}$ compared with littermate controls starting after 40 days of HFD (Figure 2A). Fat mass via EchoMRI body composition analysis (Figure 2B) was increased in female mTOR-KO ${ }^{\text {Placenta }}$ (Figure 2C) after 11 weeks of HFD. Increased food intake can contribute to obesity. However, female mTOR-KO ${ }^{\text {Placenta }}$ mice ate less relative to their body weight compared with controls (Figure 2D). We assessed whether energy expenditure could account for the obesity phenotype in female mTOR-KO ${ }^{\text {Placenta }}$ mice. Basal $\mathrm{O}_{2}$ and $\mathrm{CO}_{2}\left(\mathrm{VO}_{2}\right.$ and $\left.\mathrm{VCO}_{2}\right)$ were decreased (Figure 2, E and F), and normal respiratory exchange ratio (RER) during the day and night were observed in mTOR-KO $\mathrm{K}^{\text {Placenta }}$ compared with littermate controls (Figure 2G). Energy expenditure was also reduced in mTOR-KO ${ }^{\text {Placenta }}$ females during both day and night cycles compared with controls (Figure $2 \mathrm{H}$ ). Nonfasted hyperglycemia was also evident in mTOR-KO ${ }^{\text {Placenta }}$ compared with littermate controls (Figure 2I).

Adult female mTOR-KO Placenta mice display glucose and insulin intolerance and show deficiency in $\beta$ cell mass compensation under HFD challenge. After 6 weeks of HFD, female mTOR-KOPlacenta displayed glucose intolerance (Figure 2J) but normal insulin sensitivity at 8 weeks of HFD treatment (Figure 2K). However, a significant insulin resistance (Figure $2 \mathrm{~L}$ ) was observed at 12 weeks of HFD in mTOR-KOPlacenta compared with littermate controls. A trend toward hyperinsulinemia at 10 weeks of HFD was observed in mTOR-KO ${ }^{\text {Placenta }}$ mice $(P=$ 0.09 , Figure $2 \mathrm{M}$ ). At 13 weeks of HFD $\beta$ cell mass between mTOR-KO ${ }^{\text {Placenta }}$ and controls was normal (Figure $2 \mathrm{~N}$ ). Next we tested for $\beta$ cell function via glucose-stimulated insulin secretion (GSIS) and found that the mTOR-KO ${ }^{\text {Placenta }}$ and littermate control mice under HFD displayed similar serum insulin levels in response to high-glucose treatment (Figure 2O), and the insulin secretion stimulation index was the same (Figure 2P) under HFD treatment. Together these data suggest that mTOR-KO Placenta mice have increased susceptibility to glucose homeostasis dysfunction, in part, due to lack of $\beta$ cell mass compensation for insulin resistance. 
Female mTORKO, High-Fat Diet
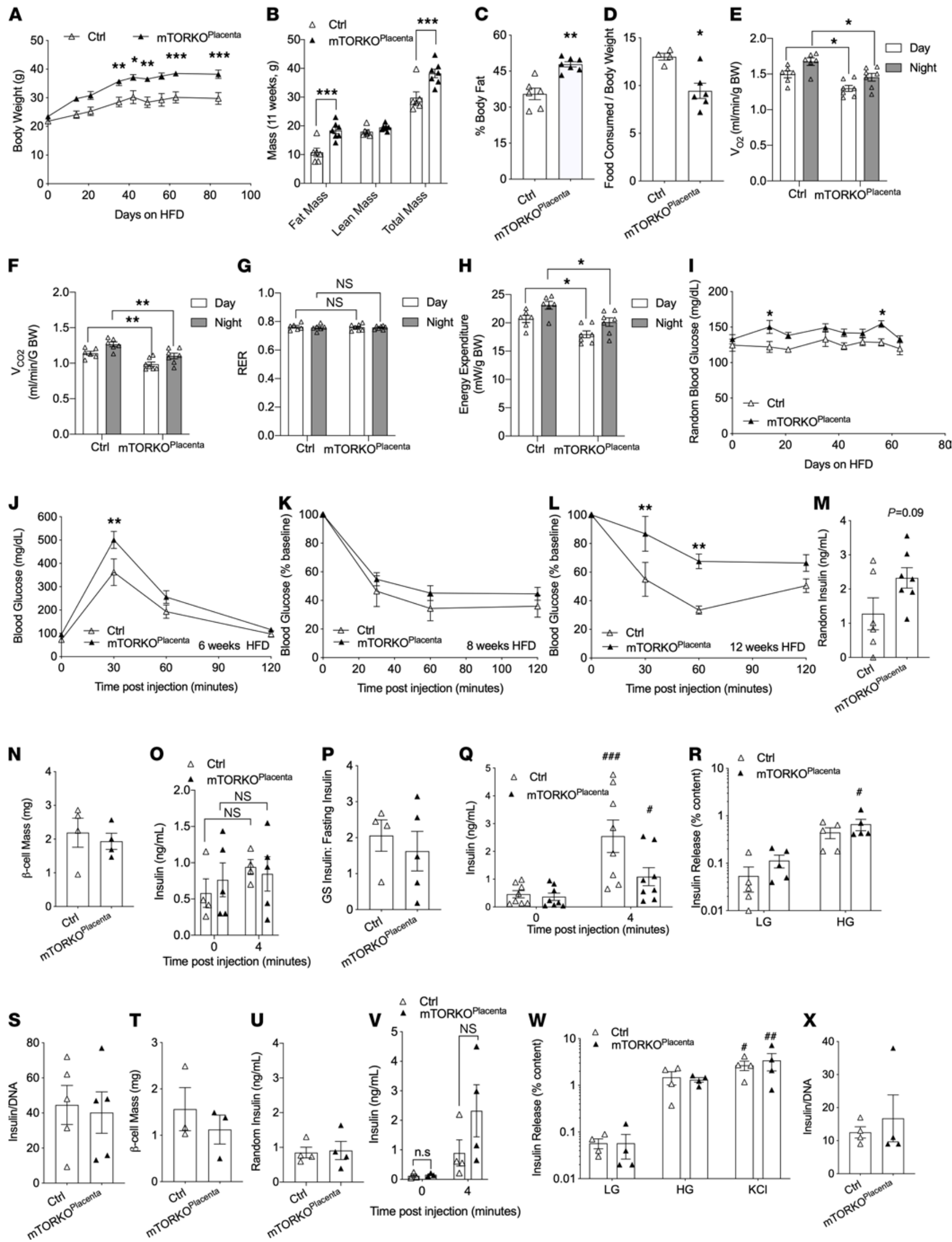
Figure 2. mTOR-KOPlacenta females become obese, glucose intolerant, and insulin resistant after HFD treatment. Weight of Ctrl versus mTOR-KOPlacenta over 90 days of HFD starting at 17 weeks of age ( $n=6,7$, respectively; A). EchoMRI body composition measurements after 11 weeks of HFD ( $n=6,7$, B) and body fat percentage (C). Food consumed per gram body weight over 48 hours $(n=4,6, \mathbf{D}) . \mathrm{VO}_{2}$ of Ctrl versus mTOR-KO Placenta at 11 weeks of HFD ( $\left.n=6, \mathbf{E}\right)$. VCO ${ }_{2}, \mathrm{RER}$, and energy expenditure $(n=6, \mathbf{F}-\mathbf{H})$. Random blood glucose of HFD $(n=6,7, \mathbf{I})$. Intraperitoneal glucose tolerance test (IPGTT) after 16 hours of fasting on HFD at 6 weeks $(n=6,7$, J). Insulin tolerance test (ITT) after 6 hours of fasting at 8 weeks of HFD ( $n=6,7, \mathbf{K})$. ITT after 6 hours of fasting at 12 weeks of HFD ( $n=6$, 7, L). Random serum insulin at 10 weeks of HFD $(n=6,7, \mathbf{M})$. $\beta$ Cell mass at 13 weeks of HFD $(n=4$ each group, $\mathbf{N})$. In vivo GSIS and secretory ratio at 10 weeks of HFD ( $n=4$ Ctrl, 5 mTOR-KOPlacenta, $\mathbf{O}$ and P). In vivo GSIS on NCD, 12 weeks old ( $n=8$ each group, $\mathbf{Q}$ ). In vitro GSIS on normal chow, 12 weeks old ( $n=5$ each group, R), and islet insulin content ( $n=5$ each group, $\mathbf{S}$ ). $\beta$ Cell mass at 12 weeks old ( $n=3$ Ctrl, 3 mTOR-KOlacenta, $\mathbf{T}$ ). Random insulin serum of normal chow at 70 weeks old ( $n=4$ each group, $\mathbf{U})$. In vivo and in vitro GSIS of normal chow 70-week-old mice ( $n=4$ each group, $\mathbf{V}$ and $\mathbf{W}$ ). Islet insulin content of normal chow 70-week-old mice ( $n=4$ each group, X). LG, low glucose; HG, high glucose. Statistical analysis was performed using 2-tailed Mann-Whitney (C, D, M, N, P, S-U, and $\mathbf{X}$ ) and 2-way ANOVA Sidak's multiple comparisons ( $\mathbf{A}, \mathbf{B}, \mathbf{E}-\mathbf{L}, \mathbf{O}, \mathbf{Q}, \mathbf{R}, \mathbf{V}$, and $\mathbf{W}$ ) with repeated measures when appropriate. Error bars represent mean \pm SEM. ${ }^{*} P<0.05,{ }^{* *} P<0.01,{ }^{* *} P<0.001$, Ctrl vs. mTOR-KO Placenta. ${ }^{*} P<0.05,{ }^{\# \#} P<0.01$, ${ }^{\# \#} P<0.001$ vs. time point 0 or LG (within genotype).

Adult female $m$ TOR-KO Placenta mice display transient reduction in $\beta$ cell function. To remove the confounding effects of HFD treatment, we assessed basal $\beta$ cell function in mTOR-KO Placenta and control female mice under NCD. In the GSIS experiment, serum insulin was reduced in mTOR-KO Placenta (Figure 2Q). However, at the islet level, in vitro GSIS in 12-week-old mice under normal chow displayed normal response to high glucose (Figure 2R). Islet insulin content (Figure 2S) and $\beta$ cell mass were found to be comparable between mTOR-KO ${ }^{\text {Placenta }}$ and controls at 12 weeks of age (Figure 2T). Additionally, $\beta$ cell function was assessed in older mice (70 weeks). Nonfasted serum insulin levels were not different between groups (Figure 2U). GSIS responses in vivo or in vitro (Figure 2, V and W) between mTOR-KO Placenta and littermate controls were comparable. Moreover, islet insulin content at this age was also normal (Figure 2X). These data suggest that the reduced serum insulin level was transient and point to the defect in extraislet tissues.Normal placental weight and fetal body weight in E17.5 embryos with gain of placental mTORC1. Similar to human studies showing association of decreased mTOR with FGR (17), the mTOR-KO ${ }^{\text {Placenta }}$ shows decreased fetal weight. However, the impact of increased placental mTOR signaling has not been studied. We hypothesized that increased mTORC1 would be associated with increased fetal weight. We tested this directly by genetically deleting TSC2, the negative regulator of mTORC1 (CYP19Cre $T S C 2^{\text {f/fl }}$, hereafter TSC2-KO ${ }^{\text {Placenta; }}$ Figure 3A). Representative images of placentas from TSC2-KO ${ }^{\text {Placenta }}$ and littermate controls are shown in Figure 3B. The JZ and LZ masses of TSC2-KO $\mathrm{KO}^{\text {Placenta }}$ were not changed in either sex (Figure 3B and Supplemental Figure 5, A-D). Male and female TSC2-KO ${ }^{\text {Placenta }}$ placental weights at E17.5 were comparable (Figure 3C). At E17.5 and P0, the fetal weight was comparable between groups (Figure 3D). The pancreas weight was decreased in male TSC2-KO Placenta at E17.5, whereas female pancreatic weight was unchanged at E17.5 and P0 (Figure 3E). Nonfasted blood glucose, insulin, $\beta$ cell mass, and pancreas insulin content were comparable between genotypes at P0 (Figure 3, F-I). Together these data show that increased mTORC1 signaling did not alter placental weight, body weight, or $\beta$ cell mass in the offspring. Although these results in neonatal pancreas were unexpected, the increased placental mTOR may still have long-term metabolic consequences in the adult offspring.

Adult male and female TSC2-KO Placenta mice show improved insulin sensitivity under NCD. To assess the impact of enhanced mTORC1 signaling during fetal development in adult offspring, we investigated glucose homeostasis status under standard diet. No difference in body weight was observed between male TSC2-KO Placenta and littermate controls (Supplemental Figure 6A). Fasting but not nonfasting blood glucose was significantly decreased between 8-week-old male TSC2-KO ${ }^{\text {Placenta }}$ and littermate controls (Supplemental Figure 6B). No difference in glucose tolerance was detected in 6- to 20-week-old males (Supplemental Figure 6C). However, improved insulin sensitivity was evident in 7- to 12-week-old male TSC2-KO Placenta compared with littermate controls (Supplemental Figure 6D). In vitro $\beta$ cell function, measured by insulin secretion in response to high glucose, $\mathrm{KCl}$, and palmitate, was comparable between primary isolated islets from control and TSC2-KO ${ }^{\text {Placenta }}$ mice (Supplemental Figure 6E). Total islet insulin content (Supplemental Figure $6 \mathrm{~F}$ ) and $\beta$ cell mass were comparable between genotypes (Supplemental Figure 6G). The female TSC2-KO $\mathrm{KO}^{\text {Placenta }}$ and littermate controls demonstrated comparable body weight gain in adulthood (Supplemental Figure $6 \mathrm{H})$. No differences were detected in fasting or nonfasting blood glucose in mice at 12-20 weeks of age (Supplemental Figure 6I). Adult female TSC2-KO $\mathrm{KO}^{\text {Placenta }}$ mice demonstrated normal glucose tolerance (Supplemental Figure 6J) and insulin sensitivity (AUC $P=0.14$, Supplemental Figure 6K).

Adult male TSC2-KO Placenta mice display improved glucose tolerance in HFD challenge. To test that enhanced mTORC1 is sufficient to improve metabolic outcomes in high-nutrient stress exposure, adult male TSC2-KO Placenta and littermate controls were put on an HFD for 10 weeks. Throughout the HFD, the male 
A

Male and Female TSC2KO Newborns

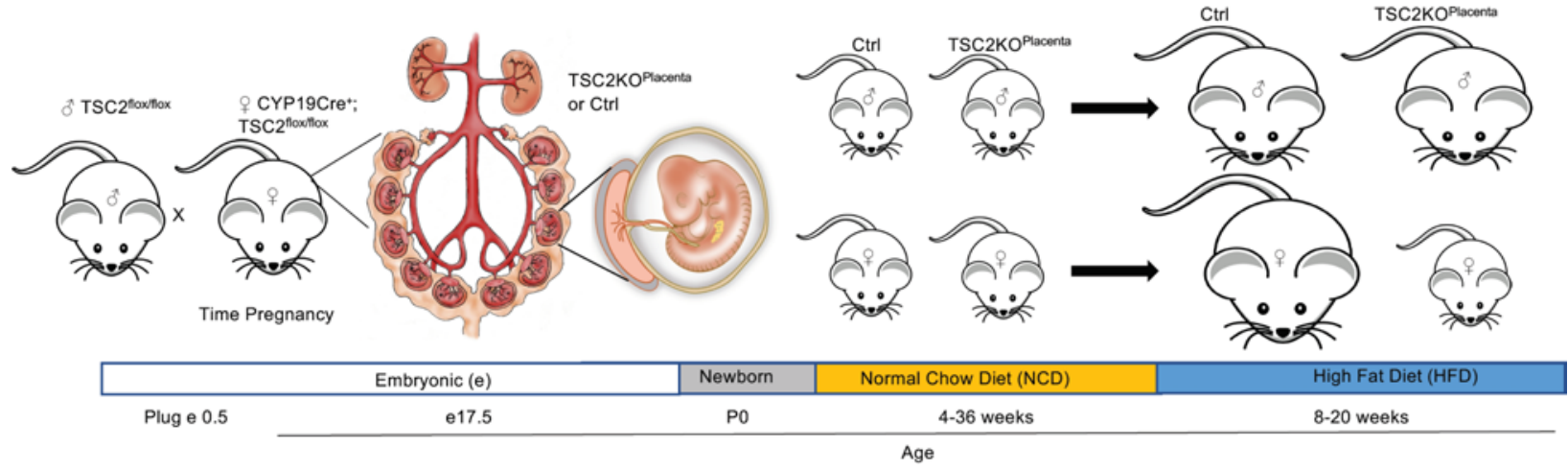

B
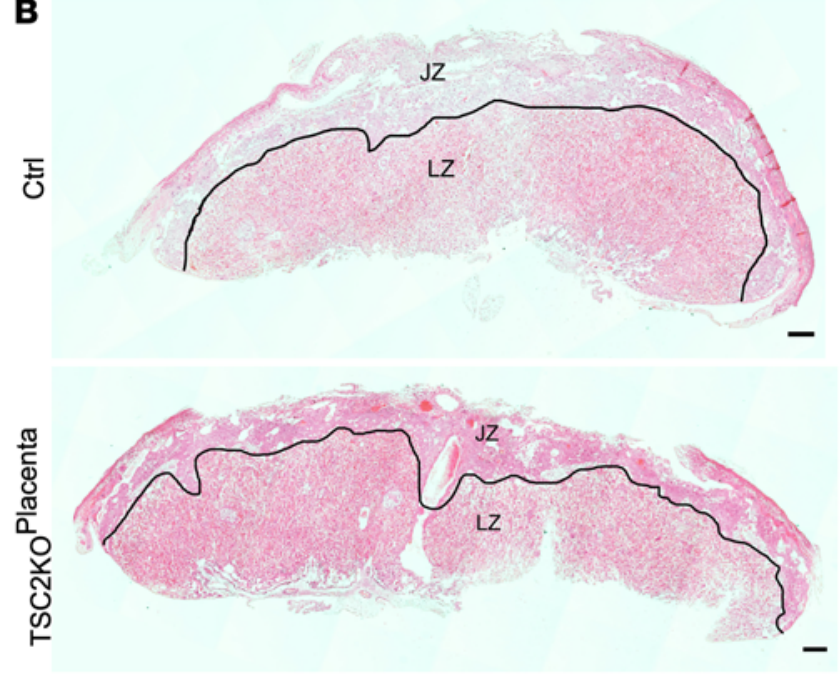

C
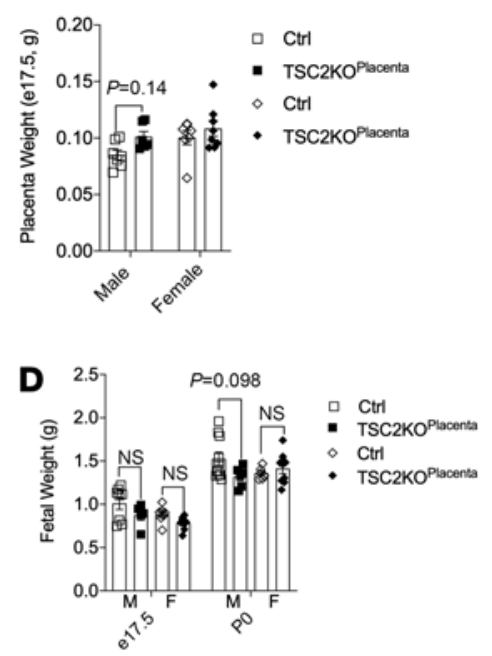
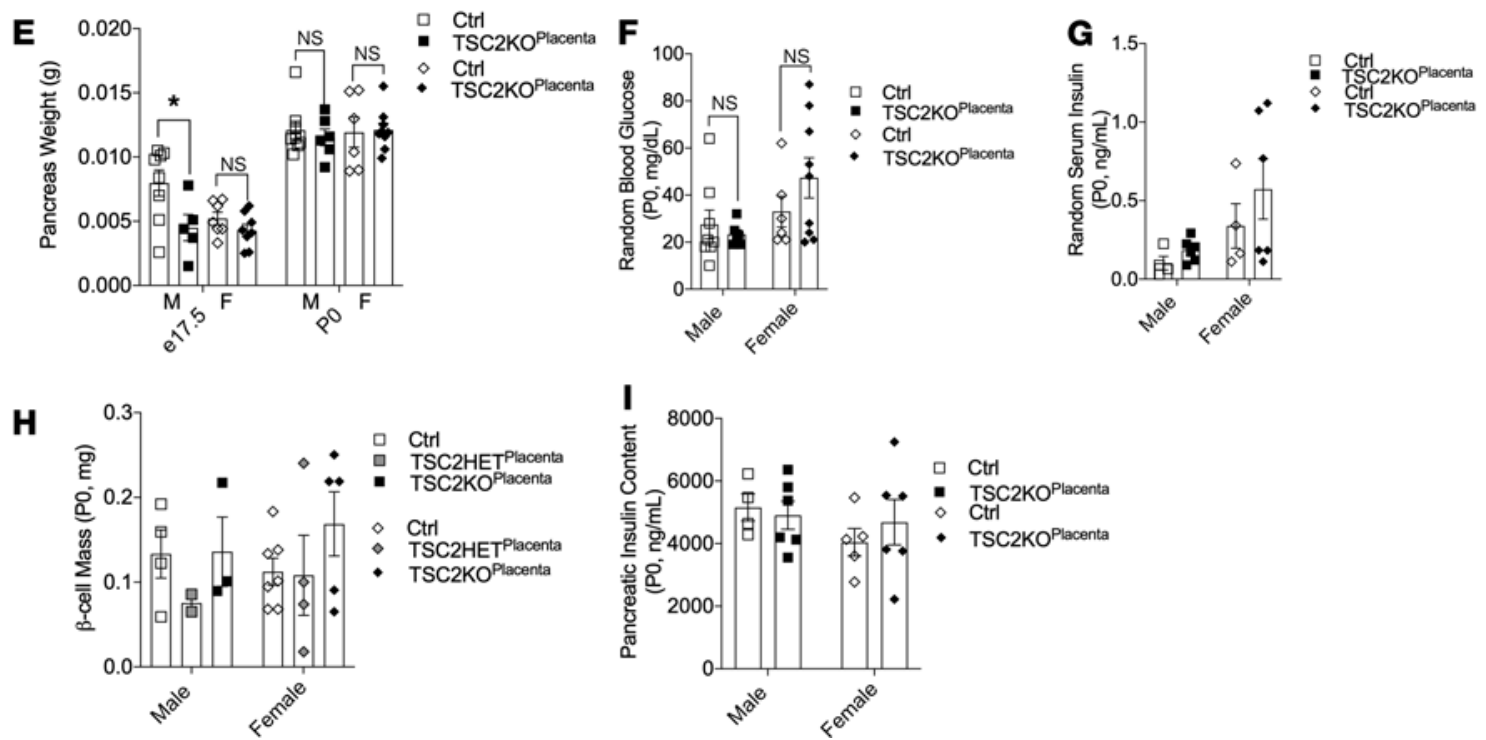

Figure 3. TSC2-KOPlacenta newborn offspring have normal placental weight and $\boldsymbol{\beta}$ cell mass. Schematic diagram and timeline of the TSC2-KO study (A). H\&E staining of E17.5 Ctrl and TSC2-KOPlacenta (B). Placental weights of male Ctrl versus TSC2-KO ${ }^{\text {Placenta }}(n=7,6)$ and female Ctrl versus TSC2-KO ${ }^{\text {Placenta }}(n=7$, 8) mice at E17.5 (C). Fetal weights of male $\left(n_{\mathrm{E} 17.5}=8,5 ; n_{\mathrm{PO}}=10,6\right)$ and female $\left(n_{\mathrm{E} 17.5}=7,8 ; n_{\mathrm{P} 0}=6,9\right)$ Ctrl vs. TSC2-KO ${ }^{\text {Placenta }}$ mice at E17.5 and PO (D). Pancreas weights of male $\left(n_{E 17.5}=8,5 ; n_{P 0}=8,6\right)$ and female $\left(n_{E 17.5}=7,8 ; n_{P 0}=6,9\right)$ mice measured at E17.5 and PO (E). Random blood glucose of males $(n=8,6)$ and females $(n=6,9)$ at PO (F). Random serum insulin of males $(n=4,6)$ and females $(n=4,6)$ at PO (C). $\beta$ Cell mass of males $(n=4$ Ctrl, 2 TSC2-HETPlacenta, 3 TSC2-KO Placenta) and females at PO ( $n=7$ Ctrl, 4 TSC2-HET Placenta, 5 TSC2-KOPlacenta, H). Whole pancreatic insulin content of males ( $n=4$ Ctrl, 6 TSC2-KO Placenta) and females ( $n=5 \mathrm{Ctrl}, 6 \mathrm{TSC2}-\mathrm{KO}^{\text {Placenta) }}$ at PO (I). Statistical analysis was performed using 2-way ANOVA with Sidak's multiple comparisons (C-I). Error bars represent mean $\pm \mathrm{SEM}$. Scale bars in images are $500 \mu \mathrm{m}$. ${ }^{*} P<0.05 \mathrm{Ctrl}$ vs. TSC2-KO Placenta. 
TSC2-KO $\mathrm{KO}^{\text {Placenta }}$ and littermate controls showed comparable body weights (Figure 4A) and food intake (Figure 4B). Nonfasted blood glucose levels were similar throughout the HFD feeding, with an exception at week 1 (Figure 4C). A trend toward improved glucose tolerance was observed in male TSC2-KO ${ }^{\text {Placenta }}$ compared with littermate controls after 2 weeks of $\operatorname{HFD}(P=0.07$, Figure 4D), suggesting a protective effect of placental mTORC1 on the adult offspring. At 2 weeks of HFD, nonfasted or fasting blood glucose levels were comparable between the groups (Figure 4E). At 4 weeks of HFD, improved glucose tolerance persisted in TSC2-KO ${ }^{\text {Placenta }}$ compared with littermate controls (Figure $4 \mathrm{~F}$ ). At this point of HFD, no difference in nonfasting or fasting blood glucose was detected between groups (Figure 4G). We tested insulin sensitivity in peripheral tissues via ITT in vivo and found no difference between male TSC2-KO ${ }^{\text {Placenta }}$ and littermate controls (Figure 4H). Moreover, the homeostatic model assessment of insulin resistance (HOMA-IR) at 6

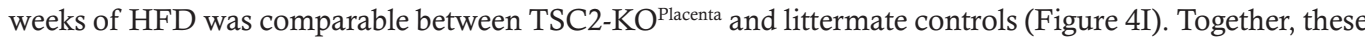
data show that adult male TSC2-KO ${ }^{\text {Placenta }}$ mice display improved glucose tolerance in HFD.

Adult male TSC2-KO Placenta mice display hypoinsulinemia and maintain $\beta$ cell function under HFD challenge. Insulin resistance is associated with hyperinsulinemia; however, we found significant hypoinsulinemia at week 4 of HFD in male TSC2-KO ${ }^{\text {Placenta }}$ compared with littermate controls (Figure 4J). Pancreatic $\beta$ cell function was assessed in vivo, and GSIS revealed a significant increase in serum insulin secretion only in TSC2-KO ${ }^{\text {Placenta }}$ mice under HFD treatment (Figure 4K). Concurrent glucose measurements during the GSIS experiment showed improved glucose tolerance in TSC2- $\mathrm{KO}^{\text {Placenta }}$ compared with littermate controls (Figure 4L). Pancreatic $\beta$ cell mass was comparable between TSC2-KO ${ }^{\text {Placenta }}$ littermate controls at 10 weeks of HFD (Figure $4 \mathrm{M}$ ). These studies suggest that TSC2- $\mathrm{KO}^{\text {Placenta }}$ males were protected from the effects of HFD on glucose tolerance by maintenance of $\beta$ cell function.

Gain of placental mTORC1 signaling confers protection from weight gain and HFD-induced glucose intolerance and insulin resistance in female mice. Next we challenged adult female TSC2-KO ${ }^{\text {Placenta }}$ and littermate controls to HFD. Unlike the male cohort, female TSC2-KO ${ }^{\text {Placenta }}$ mice demonstrated protection from body weight gain throughout the HFD (Figure 5A) associated with lower random blood glucose levels at the end of the treatment (Figure 5B). Female TSC2-KO ${ }^{\text {Placenta }}$ mice demonstrated improved glucose tolerance (Figure 5C) but normal insulin sensitivity compared with control littermates at 6 or 8 weeks of HFD, respectively (Figure 5D). At 8 weeks of HFD, nonfasting glucose levels were comparable between TSC2-KO ${ }^{\text {Placenta }}$ and littermate controls. However, fasting glucose levels were significantly reduced in TSC2-KO ${ }^{\text {Placenta }}$ mice compared with littermate controls (Figure $5 \mathrm{E}$ ).

Adult female TSC2-KO Placenta mice display hypoinsulinemia in HFD-induced obesity. Nonfasting and fasting insulin levels at 8 weeks of HFD were also significantly reduced in TSC2-KO Placenta mice compared with controls, suggesting lack of hyperinsulinemia development in these animals under HFD treatment (Figure 5F). Reduced HOMA-IR index also suggested the presence of increased insulin sensitivity in the TSC2-KO ${ }^{\text {Placenta }}$ mice compared with littermate controls (Figure 5G). Together these data suggest that increased placental mTORC1 activity conferred protection from weight gain and HFD-induced glucose and insulin intolerance.

Adult female TSC2-KO Placenta mice display normal $\beta$ cell mass but reduced function in control chow diet. Next, we aimed to identify the contribution of the pancreatic $\beta$ cell in the improved glucose and insulin tolerance in female TSC2-KO ${ }^{\text {Placenta }}$ mice. Pancreatic weight and $\beta$ cell mass were reduced in TSC2-KO ${ }^{\text {Placenta }}$ females at 12 weeks post-HFD compared with controls (Figure 5, H and I). Next, we assessed $\beta$ cell function in vivo and found that insulin secretion after glucose injection at 3 or 5 minutes reached significance relative to time 0 in both the TSC2-KO ${ }^{\text {Placenta }}$ and littermate controls in NCD (Figure 5J). At time point 3 minutes after glucose injection, however, there was a trend toward reduced insulin secretion in female TSC2-KO ${ }^{\text {Placenta }}$ compared with control mice $(P=0.09$, Figure $5 \mathrm{~J})$. We then isolated primary islets from TSC2-KO ${ }^{\text {Placenta }}$ and control female mice in NCD and performed in vitro GSIS. Insulin secretion in response to high glucose did not reach statistical significance in TSC2-KO $\mathrm{KO}^{\text {Placenta }}$ mice compared with littermate controls (Figure $5 \mathrm{~K}$ ). Insulin secretion in response to $\mathrm{KCl}$ treatment led to comparable insulin output in both the TSC2-KO ${ }^{\text {Placenta }}$ and littermate controls (Figure 5K). Additionally, islet insulin content (Figure 5L) and $\beta$ cell mass were comparable between

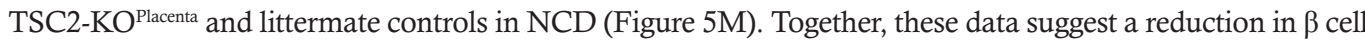
function in female offspring with TSC2-KO $\mathrm{Klacenta}^{\text {. }}$

\section{Discussion}

While placental mTOR signaling has been associated with changes in fetal growth in clinical studies, the impact of its activity on long-term metabolic health trajectory in the offspring has not been directly interrogated. Using loss- and gain-of-function mouse models, we demonstrated the impact of placental mTOR 
A

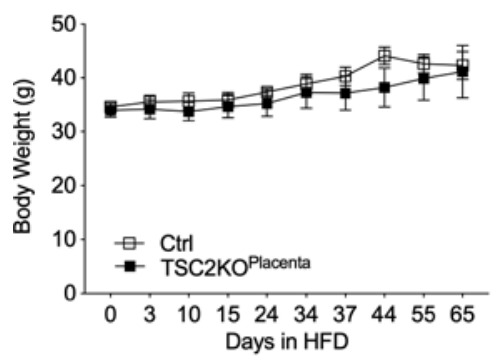

D

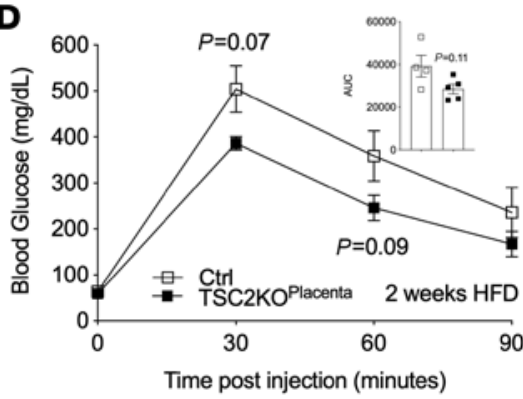

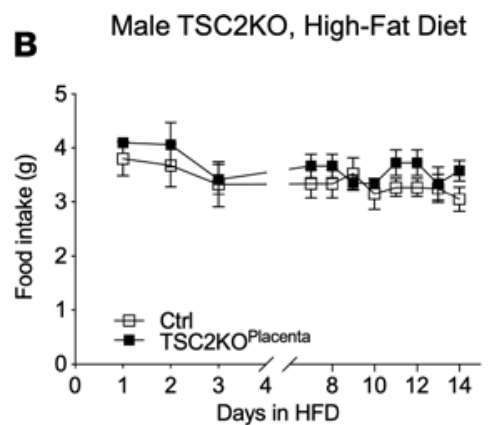
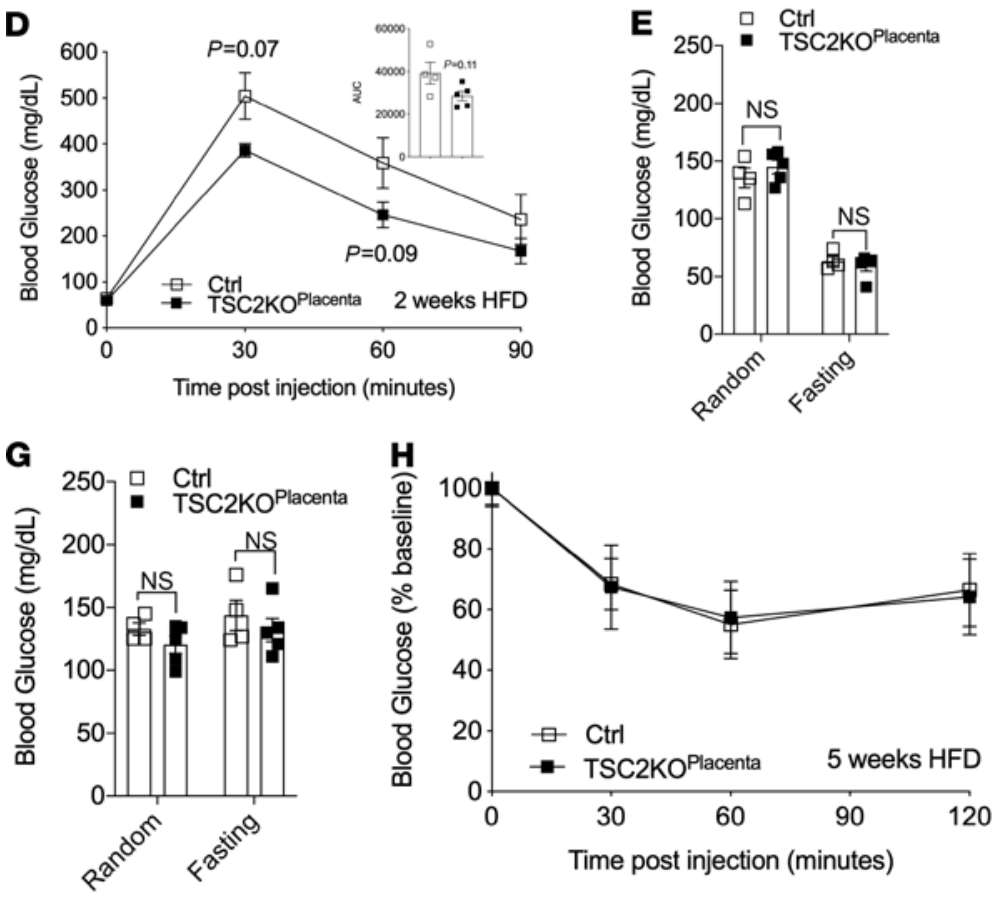

K

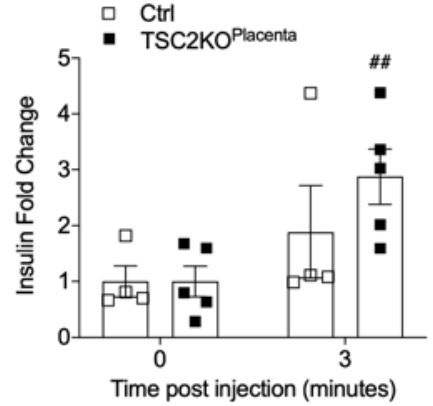

$\mathbf{L}$

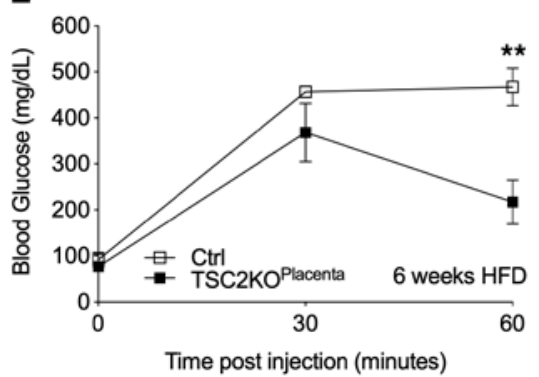

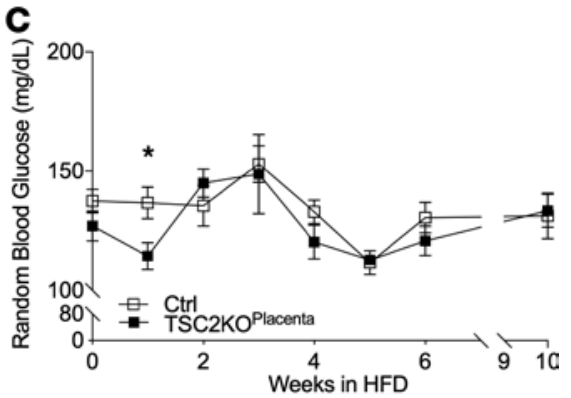

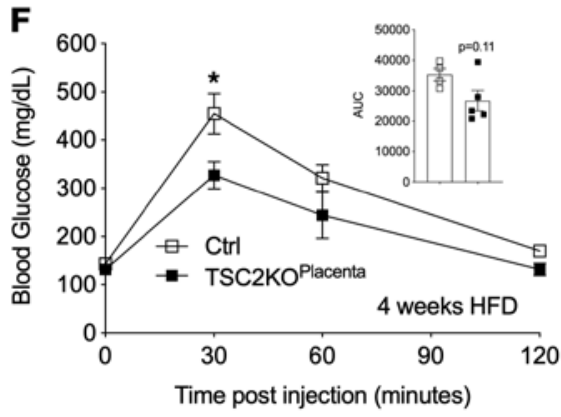

I
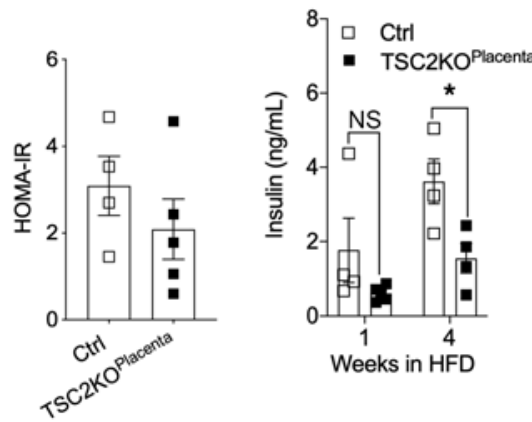

M

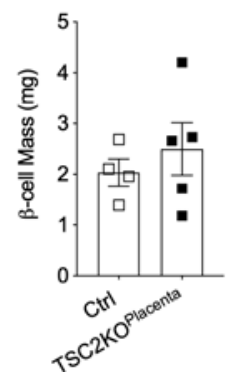

Figure 4. Adult male TSC2-KOPlacenta mice are protected from obesity-induced metabolic dysfunction. Weight progression of Ctrl versus TSC2-KO ${ }^{\text {Placenta }}$ male mice fed with HFD over 9 weeks starting at 17 weeks of age $(n=4,5$, respectively, A). Food intake measurements of HFD male mice ( $n=4,5$, B). Random blood glucose of HFD male mice $(n=4,5$, C). IPGTT after 16 hours of fasting at 2 weeks HFD $(n=4,5, \mathbf{D})$ and corresponding random and fasting blood glucose ( $n=4$, 5, E). IPGTT after 16 hours of fasting at 4 weeks of $\operatorname{HFD}(n=4,5, \mathbf{F})$ in addition to random and fasting blood glucose $(n=4,5, \mathbf{C})$. ITT at 5 weeks of HFD after 6 hours of fasting $(n=4,5, \mathrm{H})$. HOMA-IR of HFD males at 6 weeks $(n=4,5, \mathrm{I})$. Random insulin serum levels at 1 and 4 weeks of HFD ( $n=4,4-5, \mathrm{~J})$. In vivo GSIS on males at 6 weeks of HFD $(n=4,5, K)$ and concurrent IPGTT $(n=4,5, \mathrm{~L})$. $\beta$ Cell mass of males at 10 weeks of HFD $(n=4,5$, M). Statistical analysis was performed using 2-tailed Mann-Whitney (I and $\mathbf{M})$ and 2-way ANOVA with Sidak's multiple comparisons (A-H and $\mathbf{J}-\mathbf{L})$ with repeated measures when appropriate. Error bars represent mean \pm SEM. ${ }^{*} P<0.05,{ }^{* *} P<0.01$ Ctrl vs. TSC2-KO Placenta. \#\# $P<0.01$ vs. time point 0 (within genotype).

signaling on long-term metabolic health of the offspring. Using genetic models, we uncovered that offspring with loss of placental mTOR in utero displayed heightened metabolic dysfunction in response to an HFD challenge. On the other hand, enhanced placental mTORC1 in utero conferred protection from adverse metabolic dysfunction induced by HFD. With loss or gain of mTOR functional studies, we show that placental 
A
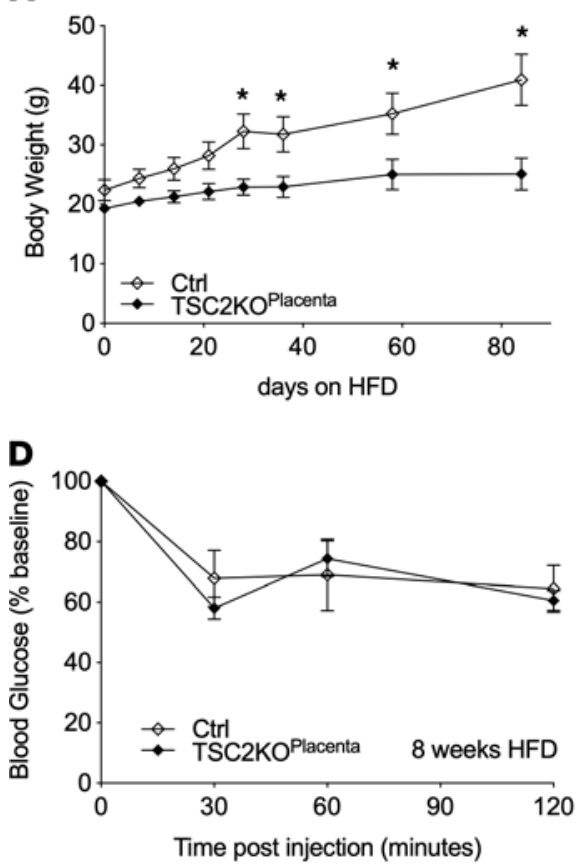

H
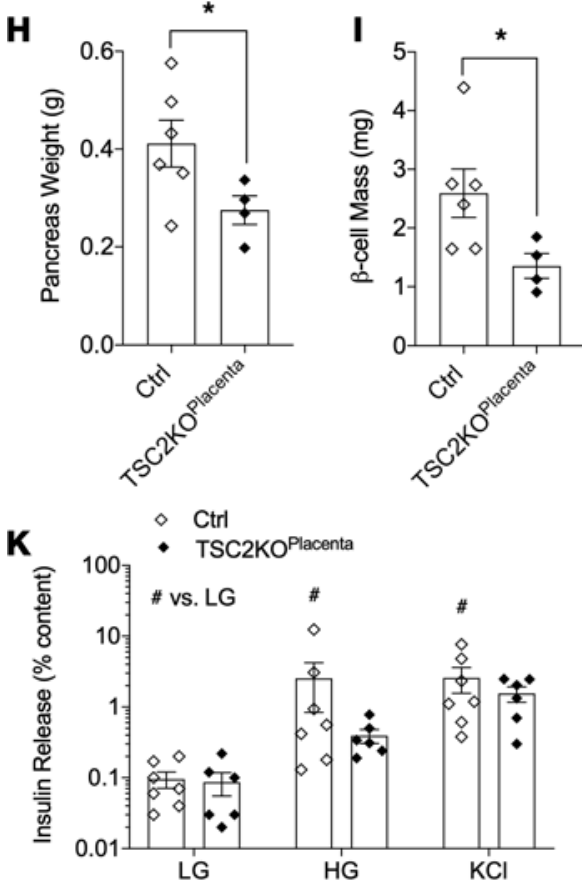

Female TSC2KO, High-Fat Diet
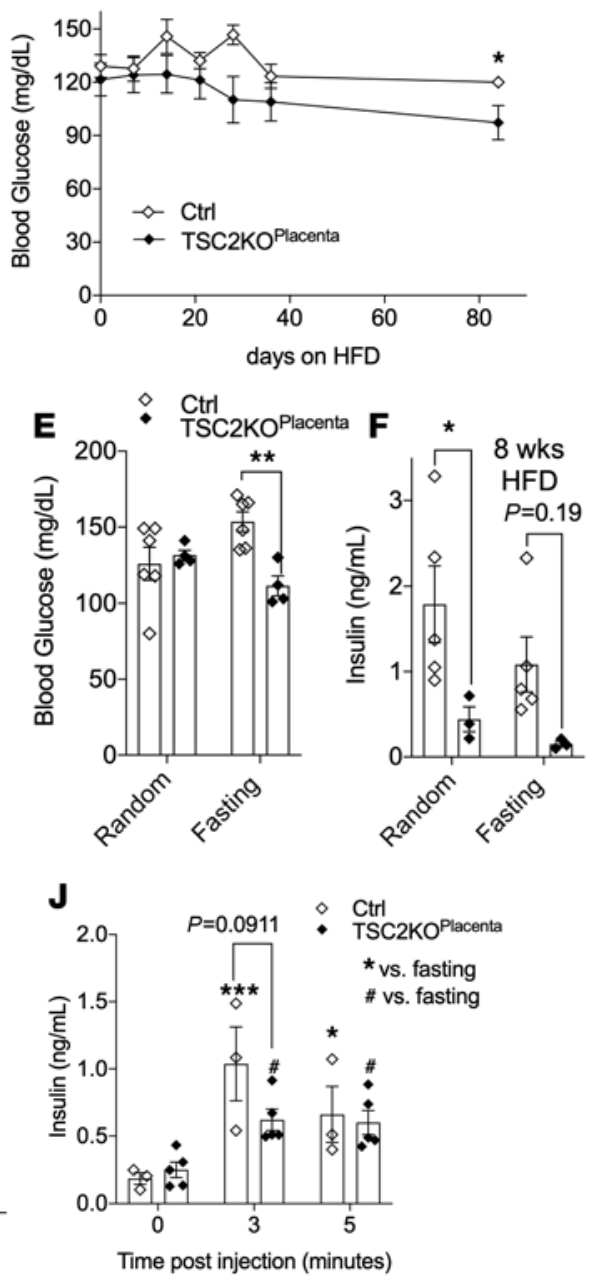
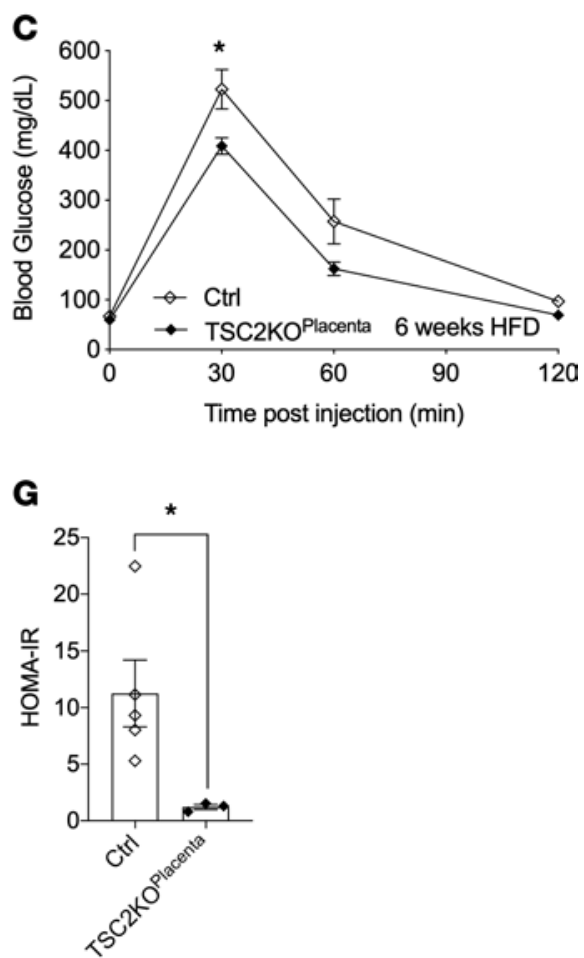
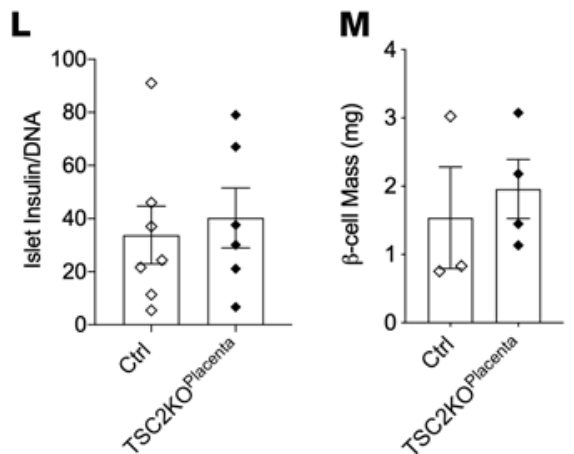

Figure 5. Adult female TSC2-KOPlacenta offspring are protected from obesity-induced metabolic dysfunction. Weight progression of female mice fed with HFD over the course of 12 weeks starting at 8 weeks old ( $n=6 \mathrm{Ctrl}, 4 \mathrm{TSC2}-\mathrm{KO} 0^{\text {Placenta }}$, A). Random blood glucose of HFD females over the duration of treatment $(n=6,4$, B). Female IPGTT after 16-hour fast at 6 weeks of HFD $(n=6,4$, C). Female ITT after 6-hour fast at 8 weeks of HFD ( $n=6$, 4, D). Blood glucose and insulin serum measurements at random and fasting times of 8-week HFD females $(n=6,4, \mathbf{E} ; n=5,3, \mathbf{F})$. HOMA-IR of HFD females at 8 weeks ( $n=5$, 3, G). Pancreas weight $(n=6,4, \mathbf{H})$ and $\beta$ cell mass $(n=6,4, \mathbf{I})$ of females at 12 weeks of HFD. In vivo GSIS of aged Ctrl versus TSC2-KOPlacenta females under normal diet $(n=3,5, \mathrm{~J})$. In vitro GSIS of 12-week-old female islets under low glucose, high glucose, high glucose plus palmitate, and $\mathrm{KCl}$ stimulation ( $n=7,6$, $\mathbf{K}$ ). Insulin content normalized to DNA $(n=7,6, \mathbf{L})$. $\beta$ Cell mass of female Ctrl versus TSC2-KO Placenta under normal chow $(n=3,4$, M). Statistical analysis was performed using 2-tailed Mann-Whitney (G-I, L, and $\mathbf{M}$ ) and 2-way ANOVA with Sidak's multiple comparisons in (A-F, J, and $\mathbf{K})$ with repeated measures when appropriate. Error bars represent mean \pm SEM. ${ }^{*} P<0.05,{ }^{* *} P<0.01,{ }^{* * *} P<0.001$ Ctrl vs. TSC2-KO Placenta. ${ }^{*} P<0.05$ vs. time point 0 or LG (within genotype). 
mTORC1 plays a major role in fetal programming of metabolic responses in the adult offspring, thereby contributing to susceptibility risk for obesity and T2D.

Placental mTOR signaling's impact on placental weight and birth weight of newborns. Enhanced placental mTOR activity has been reported in the placenta of bigger babies born to women with obesity or GDM during pregnancy (27-29). We demonstrated that disruption of mTOR (both mTORC1 and mTORC2) in the placenta resulted in smaller placental weight, whereas enhancing mTORC1 activity did not increase placental weight. Normal body weight was also observed in the offspring with increased placental mTORC1. In agreement with these data pertaining to enhanced mTORC1 activity, maternal protein supplementation that increases mTORC1 does not appear to influence birth weight (30). Increased mTORC1 activity has been correlated with higher birth weight of babies born to women with obesity (31) and GDM (28). However, it is important to note that maternal obesity or GDM has myriad effects, including but not limited to increased maternal glucose, insulin, adipokines, lipids, and low-grade metabolic inflammation, and thus, is not specific to increased mTORC1 activity per se.

Deficiency in placental mTOR in utero exacerbates responses to adverse metabolic challenge. The impact of fetal programming is often seen after an adverse exposure, or a "second hit," encountered later in life. Our data revealed that deficiency in placental mTOR in utero shaped the adult female, but not the male, offspring's adverse responses to HFD-induced obesity. Male mTOR-KO ${ }^{\text {Placenta }}$ and littermate controls responded equally in HFD-induced obesity (data not shown). On the other hand, female mTOR-KO ${ }^{\text {Placenta }}$ mice displayed a greater body weight gain throughout the course of HFD, and they displayed exacerbated adiposity and reduced energy expenditure compared with littermate controls. Female mTOR-KOPlacenta offspring exposed to HFD also demonstrated worsened glucose intolerance and increased insulin resistance compared with littermate controls. To compensate for increasing insulin demand due to insulin resistance, the pancreas responds by promoting $\beta$ cell hypersecretion and $\beta$ cell mass. A comparable $\beta$ cell mass and a trend toward increase in insulin levels between the female $\mathrm{mTOR}-\mathrm{KO}^{\text {Placenta }}$ and controls suggested insufficient compensation to combat insulin resistance present in the mTOR-KO $\mathrm{K}^{\text {Placenta }}$ offspring. The phenotypes of the mTOR-KO ${ }^{\text {Placenta }}$ mice are consistent with rodent females with increased adiposity and disrupted glucose homeostasis because of exposure to in utero undernutrition (26). The current data are also consistent with increased body mass index (BMI), waist circumference, and adiposity and disrupted lipid profile (increased cholesterol and triglycerides) seen in women and not in men (32-36). In maternal undernutrition/FGR in humans, increased BMI and adiposity have been observed in female offspring $(32,33)$. In rat and mouse models of FGR by maternal low-protein diet during the third trimester of pregnancy, male offspring develop insulin resistance associated with insufficient $\beta$ cell mass compensation $(37,38)$. Future studies focused on epigenetic changes in islets may provide mechanistic insights into fetal programming of placental mTOR.

Increased placental mTORC1 confers protection from adverse metabolic dysfunction induced by obesity. Limited data suggest that $\beta$ cell dysfunction persists in small for gestational age infants at 48 hours, but they also tend to be more sensitive to insulin (39). The relationship between insulin secretion and insulin sensitivity is hyperbolic in nature (i.e., insulin secretion decreases in response to greater insulin sensitivity) (40). Adult male and female offspring of dams fed low-protein diet during pregnancy display glucose intolerance despite increased insulin sensitivity in peripheral tissues in part due to impaired $\beta$ cell dysfunction (4). In contrast, the TSC2-KO ${ }^{\text {Placenta }}$ mice appear to have increased insulin sensitivity without glucose intolerance. Under HFD treatment, female TSC2-KO ${ }^{\text {Placenta }}$ mice were protected from gaining body weight. These animals displayed improved glucose tolerance and hypoinsulinemia with normal insulin sensitivity (tested by ITT) under HFD. Johnson et al. showed genetic evidence that a reduction of insulin level in circulation prevents HFD obesity (41), and lower circulating insulin enhances insulin sensitivity in mice (42). Lower insulin in circulation also prevents leptin-deficient Lep $o b / o b$ mice from developing obesity (43). Therefore, one possible mechanism of metabolic health protection is that lack of hyperinsulinemia contributed to the prevention of weight gain in the TSC2-KO $\mathrm{KO}^{\text {Placenta }}$ mice. It will be important for future studies to assess mechanisms of hypoinsulinemia in female TSC2-KO ${ }^{\text {Placenta }}$ mice under HFD. In addition to hypoinsulinemia, it will be important to assess the contribution of extrapancreatic tissues by sophisticated clamp studies that can help explain insulin sensitivity in the female TSC2-KO ${ }^{\text {Placenta }}$ mice.

Placental nutrient sensing by mTOR in utero and fetal programming on glucose homeostasis in the adult offspring. Low-birth weight humans and animals from preclinical models have consistently shown reduced insulin levels, and depending on the timing and severity of the insult, $\beta$ cell mass reduction can be noted in the offspring across the life span (44). The FGR in the mTOR-KO ${ }^{\text {Placenta }}$ was coupled with reduced insulin levels in 
Table 1. Metabolic phenotypes of male offspring with or without placental mTOR or TSC2

\begin{tabular}{cccc}
\hline $\begin{array}{c}\text { mTOR-KO } \\
\text { TSC2-KOacenta, }\end{array}$ Placenta & $\begin{array}{c}\text { Embryonic/Newborn } \\
\text { males }\end{array}$ & Adult NCD males & Adult HFD males \\
Body weight & Normal/Normal & Increased/Normal & Normal/Normal \\
Serum insulin & Normal/Normal & Normal & Normal/Reduced \\
GSIS in vivo & & Normal & \\
GSIS in vitro & & Normal & Normal \\
Islet insulin content & & Normal & Normal/Increased \\
$\beta$ Cell mass & Normal/Normal & Normal/Normal & Normal/Normal \\
Glucose tolerance & & Normal/Increased & \\
\hline Insulin tolerance & & & \\
\hline
\end{tabular}

the offspring serum but normal $\beta$ cell mass at P0. Increasing placental mTORC1 via TSC2 deletion also did not change basal $\beta$ cell mass or serum insulin of the offspring at birth. These data are not surprising given that gain of mTORC1 activity (also via deletion of TSC2) directly in $\beta$ cells does not increase total $\beta$ cell number in the offspring of dams with normal pregnancy (4); however, it improves $\beta$ cell mass only in the offspring of low-protein-fed dams (4). The normal glucose homeostasis phenotypes (IPGTT and ITT, summarized in Tables 1 and 2) in male and female adult mTOR-KO Placenta mice further support the lack of changes in $\beta$ cell mass in the offspring. However, these normal glucose and insulin sensitivity phenotypes under normal diet conditions are in contrast to the observed metabolic outcomes in the offspring of maternal low-protein diet-induced FGR (4) or placental insufficiency models (45-47), thus underscoring the distinct developmental programming effects of specific insults (i.e., placental mTOR vs. maternal low-protein diet or preeclampsia).

Maternal obesity increases placental mTORC1 signaling, and the associated metabolic outcomes in the offspring of obese dams are typically an increased risk for obesity and the metabolic syndrome in adulthood $(18,27)$. In the current study, gain of placenta-specific mTORC1 signaling in utero led to normal glucose tolerance and enhanced insulin sensitivity phenotypes in male and female offspring under NCD. $\beta$ Cell mass in males and females was normal. However, adult islets with increased placental mTORC1 in utero showed a significant increase in insulin secretion in response to glucose, but the amount of insulin secreted was markedly less than what was secreted by control islets. Thus, future studies should investigate whether the dampened $\beta$ cell function phenotype is intrinsic to the pancreas or a response to insulin sensitivity in peripheral tissues.

In the current study, we utilized genetic models with either loss or gain of mTOR signaling in the placenta, and we demonstrated that modulation of placental mTOR impacts long-term metabolic health trajectory of the offspring and that potential augmentation of placental mTORC1 signaling in utero may improve the health of the offspring. We observed sex differences in response to altered placental mTOR signaling, but future work will be required to understand the mechanisms. mTOR regulation of amino acid transporters $(12,25,48)$ or mitochondrial biogenesis $(49)$ in the placenta may be involved, but other unbiased analyses are still pending but necessary to fully delineate the mechanisms behind the actions of placental mTOR on

Table 2. Metabolic phenotypes of female offspring with or without placental mTOR or TSC2

\begin{tabular}{|c|c|c|c|}
\hline $\begin{array}{l}\text { mTOR-KO } \\
\text { TSC2-KOacenta, }\end{array}$ & $\begin{array}{c}\text { Embryonic/Newborn } \\
\text { females }\end{array}$ & Adult NCD females & Adult HFD females \\
\hline Body weight & Reduced/Normal & Normal/Normal & Increased/Reduced \\
\hline Serum insulin & $\begin{array}{c}\text { Reduced }(P=0.07) / \\
\text { Normal }\end{array}$ & Normal & Increased $(P=0.09) /$ Reduced \\
\hline GSIS in vivo & & Normal/Reduced & Normal \\
\hline GSIS in vitro & & Normal/Reduced & \\
\hline Islet insulin content & & Normal/Normal & \\
\hline$\beta$ Cell mass & Normal/Normal & Normal/Normal & Normal/Reduced relative to NCD \\
\hline Glucose tolerance & & Normal/Normal & Reduced/Increased \\
\hline Insulin tolerance & & Normal/Normal & Reduced/Normal \\
\hline
\end{tabular}


fetal programming of key metabolic tissues, such as the pancreas. Abnormal fetal growth affects $20 \%$ of all newborn babies around the world and increases their risk for developing obesity, diabetes, and cardiovascular disease in childhood and adulthood (50). To date, no targeted strategies have been established to treat these conditions in utero. The current data presented here advance our understanding of placental mTOR function in pregnancy complications and may provide a foundation for the development of intervention strategies for FGR and fetal overgrowth.

\section{Methods}

Generation of genetic murine models with loss or gain of placental mTOR function. Murine models were generated with loss- or gain-of-function mTOR signaling in the placenta. Trophoblast-specific Cre recombinase transgene was used and driven by the CYP19 promoter (20) with loxP-flanked sites in either $m$ TOR or TSC2 gene ( $m T O R^{f l f l}$ or TSC2 $\left.2^{f / f l}\right)$.

The offspring can be homozygous placental CYP19Cre mTOR $^{\text {flfll }}$ or CYP19Cre TSC2 $^{\text {flfl }}$ (mTOR$\mathrm{KO}^{\text {Placenta }}$ or TSC2-KO $\mathrm{KO}^{\text {Placenta)}}$ ), heterozygous CYP19Cre ${ }^{+}$TOR $^{f / W T}$ or CYP19Cre ${ }^{+}$TSC2 $^{f / W T}$ (mTOR-HET ${ }^{\text {Placenta }}$ or TSC2-HET ${ }^{\text {Placenta) }}$ ) or CYP19Cre $e^{-}$with $m T O R^{f l / f}, m T O R^{f / W T}, T S C 2^{f / f l}$, or $T S C 2^{f / W T}$ (littermate controls). As demonstrated by others, CYP19 was exclusively expressed in the placenta and not in the fetus $(20,22)$. Ai6 (CAG-ZsGreen1) mice (herein referred to as CAG) utilize a CAG system containing ZsGreen1 loxP-flanked GFP protein and are used for lineage tracing. Primers used are listed in the key resources table (Supplemental Table 1). The $m T O R^{f l / f l}, T S C 2^{f / / f}$, and Ai6 mice were purchased from The Jackson Laboratory. CYP19Cre mice were donated by G. Leone from Ohio State University. All mice were generated on a mixed background and group housed on a 14-hour light/10-hour dark cycle with ad libitum access to standard diet or HFD (D12492, 60\% kcal fat, Research Diets Inc) starting at the age where indicated. For rigor, sex was considered as an independent variable, and data were segregated and analyzed separately.

Pancreas section collection and $\beta$ cell mass analysis in animals. Time pregnancies were set up and observation of a plug were marked as embryonic day 0.5 . At E17.5 or E18.5, the dam was sacrificed, and the pancreas was harvested from the fetus. Newborn pancreata were collected at birth (P0). Harvested newborn and embryonic pancreata were soaked in $3.75 \%$ formalin for 5 hours, or overnight for adult pancreata, then in $70 \%$ ethanol prior to embedding. Adult tissue blocks were sectioned at $5 \mu \mathrm{m}$ thickness until $50 \mu \mathrm{m}$ of tissue was collected, then removed $200 \mu \mathrm{m}$ from the block, repeating until 5 different areas of the pancreas were collected. Newborn or embryonic tissue blocks were collected from top to bottom at $5 \mu \mathrm{m}$ thickness. Analysis of $\beta$ cell mass was previously described (51).

Immunostaining and histology quantification. Immunofluorescence and IHC techniques were utilized in paraffin-embedded mouse or human tissues as previously described (52). H\&E was used to assess macroscopic observation of the mouse placenta. Sagittal cuts were performed on the middle section of murine placenta and paraffin-embedded cut side down. The standard Citri Solv (Thermo Fisher Scientific) deparaffinization and dehydration procedure was done on both mouse and human tissue. Both H\&E and IHC staining were performed per manufacturer's protocol. All murine samples were visualized using Nikon ECLIPSE Ni-E microscope. Quantification of chromogen immunostaining was analyzed using ImageJ (NIH, http://imagej. nih.gov/ij/). See Supplemental Table 1 for key resources information.

Tissue clearing and microscopy of newborn animals. The tissue was fixed in fresh $4 \%$ paraformaldehyde in $0.1 \mathrm{M}$ PBS, $\mathrm{pH} 7.2$, for 24 hours. Then the fixed tissue was cleared using the Logos Biosystems X-CLARITY System as previously described (53). Images were taken using the Ni-E microscope with $\mathrm{C} 2+$ si confocal microscope in spectral mode utilizing GaAsP spectral detectors by using the Nikon 10× glycerol, $0.5 \mathrm{NA}, 5.5 \mathrm{~mm}$ WD objective (Nikon Instruments) while using the $488 \mathrm{~nm}$ laser and collecting emission spectra from 500-650 $\mathrm{nm}$ at $10 \mathrm{~nm}$ spectral resolution. Tiled, Z-series images were spectrally unmixed using EGFP library spectra and processed in Nikon Elements v5.01. The tissue clearing and microscopy were performed in the University Imaging Centers, University of Minnesota (https://med.umn.edu/uic).

Primary mouse islet isolation, Western blot, insulin secretion in vitro, and glucose and insulin levels. Islets were isolated by collagenase digestion through the common duct as previously described (54). After handpicking the islets and allowing an overnight rest period, the islets were prepped for in vitro glucose stimulus insulin secretion assays as described (54). Protein samples were prepared from 3 to 4 pancreata, livers, and inguinal fat pads from each treatment group. Samples were flash frozen with liquid nitrogen and then homogenized. Samples were washed with $1 \times$ PBS prior to incubating in lysis buffer (Cell Signaling Technology) with protease inhibitor and phosphatase inhibitor cocktails (Roche Applied Science). Western blot preparation, procedure, 
and densitometry analysis were done as previously described (51). See Supplemental Table 1 for primary antibodies used. Glucose (detected by glucose meter) and insulin (assessed by ELISA) were measured from trunk blood collected from P0 animals as previously described (51).

$A A$ analysis and leucine placental perfusion experiment. Serum AAs from P0 (collected from trunk blood) were measured by simultaneous liquid chromatography-mass spectrometry (LC-MS) as previously described (55). Briefly, samples were derivatized with dansyl chloride (DC) prior to the LC-MS analysis. A total of $5 \mu \mathrm{L}$ of sample or standard was mixed with $5 \mu \mathrm{L}$ of $100 \mu \mathrm{M}$ p-chlorophenylalanine (internal standard), $50 \mu \mathrm{L}$ of 10 $\mathrm{mM}$ sodium carbonate, and $100 \mu \mathrm{L}$ of $\mathrm{DC}\left(3 \mathrm{mg} / \mathrm{mL}\right.$ in acetone). The mixture was incubated at $60^{\circ} \mathrm{C}$ for 15 minutes and centrifuged at $18,000 \mathrm{~g}$ for 10 minutes. The supernatant was transferred into an HPLC vial (Thermo Fisher Scientific) for LC-MS analysis. Unidirectional flow from materno-fetal placental interface was measured using radiolabeled isotopes as previously described (56). At gestation day 17.5, the maternal jugular vein was exposed under isoflurane anesthesia. A total of $100 \mu \mathrm{Ci}$ of ${ }^{3} \mathrm{H}$-Leucine was injected through a jugular catheter. Blood was collected at time points 0 and 10 minutes via tail vein before and after injection, respectively, for count readings to ensure successful injection. After 10 minutes, the dam was euthanized. The placenta and fetus (postdecapitation) were placed in scintillation tubes with Biosol and incubated overnight in a water bath at $95^{\circ} \mathrm{C}$ for digestion. The resulting lysates were diluted $1: 10$ with Bioscint and then ${ }^{3} \mathrm{H}$ counted with liquid scintillation counter (Beckman Coulter, LS6500).

Metabolic phenotyping. Glucose and insulin tolerance tests were performed by intraperitoneal delivery of $2 \mathrm{~g} / \mathrm{kg}$ glucose or $0.75 \mathrm{U} / \mathrm{kg}$ insulin (Novolin, Novo Nordisk Inc) to mice after 12 or 6 hours of fasting as previously described (57). The HOMA-IR index was calculated as (fasting insulin $[\mathrm{ng} / \mathrm{mL}] \times$ fasting glucose $[\mathrm{mmol} / \mathrm{L}]) / 22.5$ to assess insulin resistance. Metabolic cages for whole-animal energy expenditure (Oxymax/CLAMS Lab Animal Monitoring System, Columbus Instruments) and body composition via EchoMRI (Echo Medical Systems LLC) service were provided by the University of Minnesota Integrative Biology and Physiology Core.

Statistics. Data were analyzed by using Mann-Whitney $t$ test (2 tailed) or 2-way ANOVA with Sidak's multiple comparisons test as indicated in the legends. A $P$ value of less than 0.05 was considered statistically significant. Data were evaluated by GraphPad Prism 7 or 8 (GraphPad Software).

Study approval. All animal studies were approved by the Institutional Animal Care and Use Committee (protocol 1806-36072A) at the University of Minnesota, and radiation studies were approved by the University Health and Safety Department of Radiation Safety at the University of Minnesota.

\section{Author contributions}

BA, DCB, SJ, ADL, MB, AW, TZ, RM, MS, DLF, and EUA designed experiments, generated and analyzed data, assisted with manuscript preparation, and approved the final version; BA and EUA interpreted the data and wrote and edited the manuscript; MRBPC contributed to the discussion; and EUA conceived the study, acquired funding, was in charge of overall direction of this work, and is the guarantor of this work.

\section{Acknowledgments}

We thank Robert Sorenson (University of Minnesota), Cyprian Weaver (University of Minnesota), David Bernlohr (University of Minnesota), Brigid Gregg (University of Michigan), Jean Regal (University of Minnesota-Duluth), and Sarah Wernimont (University of Minnesota) for discussion; Mark Sanders and Thomas Pengo (University of Minnesota University Imaging Centers) for imaging expertise; and G. Leone (The Ohio State University) for the donation of the CYP19Cre mice. Microscope and imaging support was provided by the Department of Integrative Biology and Physiology (IBP) and the University Imaging Centers (https://med. umn.edu/uic). Body composition analysis and whole-animal energy expenditure were serviced by the IBP Core. We thank Pilar Guzman for assistance with surgery. We also thank Chi Chen for his assistance in measuring AAs (University of Minnesota). This work was supported by NIH grants (R21DK112144, R21HD100840, R03DK114465, R01DK115720), Regenerative Medicine Minnesota, and IBP startup funds to EUA; Philippine Council for Health Research and Development - Department of Science and Technology (grant MOA 18-0200 and 19-0663) to MRBPC; NIH T32 DK007203 to MB; and NIH R03DK11446501AIS1 to BA.

Address correspondence to: Emilyn Uy Alejandro, University of Minnesota, CCRB, \#3-142, 2231 6th St. SE, Minneapolis, Minnesota 55455, USA. Phone: 612.301.7685; Email: ealejand@umn.edu. 
1. Danaei G, et al. National, regional, and global trends in fasting plasma glucose and diabetes prevalence since 1980: systematic analysis of health examination surveys and epidemiological studies with 370 country-years and 2.7 million participants. Lancet. 2011;378(9785):31-40

2. Lango $\mathrm{H}$, et al. Assessing the combined impact of 18 common genetic variants of modest effect sizes on type 2 diabetes risk Diabetes. 2008;57(11):3129-3135.

3. Morris AP, et al. Large-scale association analysis provides insights into the genetic architecture and pathophysiology of type 2 diabetes. Nat Genet.2012;44(9):981-990.

4. Alejandro EU, et al. Maternal diet-induced microRNAs and mTOR underlie beta cell dysfunction in offspring. J Clin Invest. 2014;124(10):4395-4410.

5. Sandovici I, et al. Developmental and environmental epigenetic programming of the endocrine pancreas: consequences for type 2 diabetes. Cell Mol Life Sci. 2013;70(9):1575-1595.

6. Hales CN, Barker DJ. Type 2 (non-insulin-dependent) diabetes mellitus: the thrifty phenotype hypothesis. Diabetologia. 1992;35(7):595-601

7. Martin J, et al. Births: final data for 2018. Natl Vital Stat Rep. 2019;68(13):1-47.

8. Mohan R, et al. Fetal undernutrition, placental insufficiency and pancreatic beta-cell development programming in utero. Am $J$ Physiol Regul Integr Comp Physiol. 2018;315(5):R867-R878.

9. Dimasuay KG, et al. Placental responses to changes in the maternal environment determine fetal growth. Front Physiol. $2016 ; 7: 12$.

10. Roos S, et al. Mammalian target of rapamycin in the human placenta regulates leucine transport and is down-regulated in restricted fetal growth. J Physiol. 2007;582(pt 1):449-459.

11. Jansson T, et al. The emerging role of mTORC1 signaling in placental nutrient-sensing. Placenta. 2012;33(suppl 2):e23-e29.

12. Rosario FJ, et al. Regulation of amino acid transporter trafficking by mTORC1 in primary human trophoblast cells is mediated by the ubiquitin ligase Nedd4-2. Clin Sci (Lond). 2016;130(7):499-512.

13. Saha S, et al. MicroRNA-141-3p and miR-200a-3p regulate insulin-like growth factor 2 during mouse placental development. Mol Cell Endocrinol. 2015;414:186-193.

14. Laviola L, et al. Intrauterine growth restriction in humans is associated with abnormalities in placental insulin-like growth factor signaling. Endocrinology. 2005;146(3):1498-1505.

15. Bhasin KK, et al. Maternal low-protein diet or hypercholesterolemia reduces circulating essential amino acids and leads to intrauterine growth restriction. Diabetes. 2009;58(3):559-566.

16. Kavitha JV, et al. Down-regulation of placental mTOR, insulin/IGF-I signaling, and nutrient transporters in response to maternal nutrient restriction in the baboon. FASEB J. 2014;28(3):1294-1305.

17. Roos S, et al. Placental mTOR links maternal nutrient availability to fetal growth. Biochem Soc Trans. 2009;37(pt 1):295-298.

18. Rosario FJ, et al. Activation of placental insulin and mTOR signaling in a mouse model of maternal obesity associated with fetal overgrowth. Am J Physiol Regul Integr Comp Physiol. 2016;310(1):R87-R93.

19. Alejandro EU, et al. Gestational diabetes mellitus: a harbinger of the vicious cycle of diabetes. Int J Mol Sci. 2020;21(14):5003

20. Wenzel PL, Leone G. Expression of Cre recombinase in early diploid trophoblast cells of the mouse placenta. Genesis. 2007;45(3):129-134.

21. Howerton CL, Bale TL. Targeted placental deletion of OGT recapitulates the prenatal stress phenotype including hypothalamic mitochondrial dysfunction. Proc Natl Acad Sci U S A. 2014;111(26):9639-9644.

22. Lopez-Tello J, et al. Fetal and trophoblast PI3K p110 $\alpha$ have distinct roles in regulating resource supply to the growing fetus in mice. Elife. 2019;8:e45282.

23. Elghazi L, et al. Role of nutrients and mTOR signaling in the regulation of pancreatic progenitors development. Mol Metab. 2017;6(6):560-573.

24. Rosario FJ, et al. Maternal protein restriction in the rat inhibits placental insulin, mTOR, and STAT3 signaling and down-regulates placental amino acid transporters. Endocrinology. 2011;152(3):1119-1129.

25. Rosario FJ, et al.Mammalian target of rapamycin signalling modulates amino acid uptake by regulating transporter cell surface abundance in primary human trophoblast cells. J Physiol. 2013;591(3):609-625.

26. Dearden L, et al. Sex and gender differences in developmental programming of metabolism. Mol Metab. 2018;15:8-19.

27. Jansson N, et al. Activation of placental mTOR signaling and amino acid transporters in obese women giving birth to large babies. J Clin Endocrinol Metab. 2013;98(1):105-113.

28. Shang M, Wen Z. Increased placental IGF-1/mTOR activity in macrosomia born to women with gestational diabetes. Diabetes Res Clin Pract. 2018;146:211-219.

29. Sati L, et al. Expression of mammalian target of rapamycin and downstream targets in normal and gestational diabetic human term placenta. Reprod Sci. 2016;23(3):324-332.

30. Rush D, et al. Controlled trial of prenatal nutrition supplementation defended. Pediatrics. 1980;66(4):56-658.

31. Howell KR, Powell TL. Effects of maternal obesity on placental function and fetal development. Reproduction. 2017;153(3):R97-R108

32. Ravelli AC, et al. Obesity at the age of $50 \mathrm{y}$ in men and women exposed to famine prenatally. Am J Clin Nutr. 1999;70(5):811-816

33. Stein $\mathrm{AD}$, et al. Anthropometric measures in middle age after exposure to famine during gestation: evidence from the Dutch famine. Am J Clin Nutr.2007;85(3):869-876.

34. Roseboom TJ, et al. Effects of prenatal exposure to the Dutch famine on adult disease in later life: an overview. Mol Cell Endocrinol. 2001;185(1-2):93-98.

35. Lumey LH, et al. Lipid profiles in middle-aged men and women after famine exposure during gestation: the Dutch Hunger Winter Families Study. Am J Clin Nutr. 2009;89(6):1737-1743.

36. Kuo AH, et al. Sex-dimorphic acceleration of pericardial, subcutaneous, and plasma lipid increase in offspring of poorly nourished baboons. Int J Obes (Lond).2018;42(5):1092-1096.

37. Alejandro EU, et al. Maternal low-protein diet on the last week of pregnancy contributes to insulin resistance and beta-cell dysfunction in the mouse offspring. Am J Physiol Regul Integr Comp Physiol. 2020;319(4):R485-R496.

38. Venci RO, et al. Malnutrition during late pregnancy exacerbates high-fat-diet-induced metabolic dysfunction associated with lower sympathetic nerve tonus in adult rat offspring. Nutr Neurosci. 2020;23(6):432-443. 
39. Bazaes RA, et al. Glucose and lipid metabolism in small for gestational age infants at 48 hours of age. Pediatrics. 2003;111(4 pt 1):804-809.

40. Kahn SE, et al. Quantification of the relationship between insulin sensitivity and beta-cell function in human subjects. Evidence for a hyperbolic function. Diabetes. 1993;42(11):1663-1672.

41. Mehran AE, et al. Hyperinsulinemia drives diet-induced obesity independently of brain insulin production. Cell Metab. 2012;16(6):723-737

42. Templeman NM, et al. Reduced circulating insulin enhances insulin sensitivity in old mice and extends lifespan. Cell Rep. 2017;20(2):451-463.

43. D'Souza AM, et al. Suppressing hyperinsulinemia prevents obesity but causes rapid onset of diabetes in leptin-deficient Lep(ob/ ob) mice. Mol Metab. 2016;5(11):1103-1112.

44. Boehmer BH, et al. The impact of IUGR on pancreatic islet development and beta-cell function. J Endocrinol. 2017;235(2):R63-R76.

45. Akhaphong B, et al. Reduced uterine perfusion pressure causes loss of pancreatic $\beta$ cell area but normal function in fetal rat offspring. Am J Physiol Regul Integr Comp Physiol. 2018;315(6):R1220-R1231.

46. Heltemes A, et al. Chronic placental ischemia alters amniotic fluid milieu and results in impaired glucose tolerance, insulin resistance and hyperleptinemia in young rats. Exp Biol Med (Maywood). 2010;235(7):892-899.

47. Simmons RA, et al. Intrauterine growth retardation leads to the development of type 2 diabetes in the rat. Diabetes. 2001;50(10):2279-2286.

48. Chen YY, et al. Increased ubiquitination and reduced plasma membrane trafficking of placental amino acid transporter SNAT-2 in human IUGR. Clin Sci (Lond). 2015;129(12):1131-1141.

49. Rosario FJ, et al. Mechanistic target of rapamycin complex 1 promotes the expression of genes encoding electron transport chain proteins and stimulates oxidative phosphorylation in primary human trophoblast cells by regulating mitochondrial biogenesis. Sci Rep. 2019;9(1):246.

50. Gluckman PD, et al. Effect of in utero and early-life conditions on adult health and disease. NEngl J Med. 2008;359(1):61-73.

51. Baumann D, et al. Role of nutrient-driven O-GlcNAc-posttranslational modification in pancreatic exocrine and endocrine islet development. Development. 147(7):dev186643.

52. Lockridge $\mathrm{AD}$, et al. Serine racemase is expressed in islets and contributes to the regulation of glucose homeostasis. Islets. 2016;8(6):195-206.

53. Lyons CE, et al. Optogenetic-induced sympathetic neuromodulation of brown adipose tissue thermogenesis. FASEB J. 2020;34(2):2765-2773

54. Lockridge A, et al. Islet O-GlcNAcylation is required for lipid potentiation of insulin secretion through SERCA2. Cell Rep. 2020;31(5):107609.

55. Ma Y, et al. Metabolomic evaluation of scenedesmus sp. as a feed ingredient revealed dose-dependent effects on redox balance, intermediary and microbial metabolism in a mouse model. Nutrients. 2019;11(9):1971.

56. Coan PM, et al. Adaptations in placental phenotype support fetal growth during undernutrition of pregnant mice. $J$ Physiol. 2010;588(pt 3):527-538

57. Jo S, et al. Translational factor eIF4G1 regulates glucose homeostasis and pancreatic $\beta$-cell function. Diabetes. 2021;70(1):155-170. 